LBL-36963

UC-401

\title{
Liquid-Liquid Equilibria for Polymer Solutions and Blends, Including Copolymers
}

\author{
Yuhua Song, Toshiaki Hino, Stephen M. Lambert, \\ and John M. Prausnitz \\ Department of Chemical Engineering \\ University of California, Berkeley \\ and \\ Chemical Sciences Division \\ Lawrence Berkeley Laboratory \\ University of California \\ Berkeley, California 94720
}

February 1995

\section{DISCLAIMER}

This report was prepared as an account of work sponsored by an agency of the United States Government. Neither the United States Government nor any agency thereof, nor any of their employees, makes any warranty, express or implied, or assumes any legal liability or responsibility for the accuracy, completeness, or usefulness of any information, apparatus, product, or process disclosed, or represents that its use would not infringe privately owned rights. Reference herein to any specific commercial product, process, or service by trade name, trademark, manufacturer, or otherwise does not necessarily constitute or imply its endorsement, recommendation, or favoring by the United States Government or any agency thereof. The views and opinions of authors expressed herein do not necessarily state or reflect those of the United States Government or any agency thereof.

This work was supported by the Director, Office of Energy Research, Office of Basic Energy Sciences, Chemical Sciences Division, of the U.S. Department of Energy under Contract No. DE-AC03-76SF00098. 


\section{DISCLAIMER}

Portions of this document may be illegible in electronic image products. Images are produced from the best available original document. 


\title{
Liquid-liquid equilibria for polymer solutions and blends, including copolymers
}

\author{
Yuhua Song*, Toshiaki Hino, Stephen M. Lambert ${ }^{+}$, and John M. Prausnitz \\ Department of Chemical Engineering, University of California, Berkeley, and Chemical Sciences Division, \\ Lawrence Berkeley Laboratory, University of California, Berkeley, CA 94720
}

\begin{abstract}
A simplified perturbed hard-sphere-chain (PHSC) theory is applied to interpret, correlate, and (in part) predict liquid-liquid equilibria (LLE) for polymer solutions and blends, including copolymers. The PHSC equation of state uses a hard-sphere-chain reference system plus a van der Waals attractive perturbation. Three pure-component parameters are obtained from readily available thermodynamic properties. Mixture parameters are obtained using pure-component parameters, conventional combining rules, and one or two binary constants. Theoretical and experimental coexistence curves and miscibility maps show good agreement for selected blends containing polymers and copolymers. For LLE of dilute or semi-dilute solvent/polymer solutions, it is necessary to decrease the pure-component polymer chain length, probably because the mean-field approximation is not suitable for such solutions.
\end{abstract}

\section{INTRODUCTION}

Equations of state (EOS) provide useful tools for correlating and estimating thermodynamic properties of polymer solutions [1,2].The EOS theory has successfully explained both lower-critical-solution-temperature (LCST) and upper-critical-solution-temperature (UCST) phenomena for polymer solutions, in contrast to the classical Flory-Huggins polymer-solution theory [3] that fails to describe LCST behavior at elevated temperatures.

Recently, we presented a perturbed hard-sphere-chain (PHSC) EOS applicable to mixtures containing solvents, polymers, and copolymers [4-9]. We first developed a hard-sphere-chain (HSC) equation of state by generalizing Chiew's result for HSC mixtures through the Carnahan-Starling radial distribution function for HSC mixtures at contact [5]. For real fluids, a van der Waals perturbation was added to the reference HSC EOS and the Song-Mason [10] method was used to scale the van der Waals covolume $b$ and the attractive energy parameter $a$ in terms of two pair-potential parameters. In this method, two universal functions of a reduced temperature were introduced to represent the effect of temperature on $b$ and $a$ [6]; they were determined from the vapor pressures and the densities of saturated liquid and vapor of argon and methane and extended to molecular fluids and polymers by using a temperature scaling factor $s$.

For mixtures containing polymers and copolymers, no mixing rules are required for the hard-sphere-chain contribution. Only the perturbation needs the van der Waals one-fluid ( $v d W 1$ ) mixing theory [4,7-9]. The PHSC EOS can reproduce all types of fluid phase equilibria that have been found experimentally in binary and ternary mixtures containing polymers and copolymers [7-9]. It can also quantitatively describe UCST and LCST behavior and miscibility maps in mixtures containing polymers and copolymers $[4,7,9]$.

In this paper, we present a simplified version of the PHSC EOS. The scaling factor $s$ is removed and the two universal functions are redetermined from thermodynamic properties of argon and methane over a wider range of fluid temperatures and densities. Removal of $s$ allows use of simpler combining rules for mixtures. For pure fluids and polymers, the new PHSC EOS is still characterized by three molecular parameters: segment number per molecule $r$, segment size $\sigma$, and non-bonded segment-segment interaction energy $\varepsilon$; they can be obtained from

\footnotetext{
* Present address: Simulation Sciences, Inc., 601 South Valencia Avenue, Brea, CA 92621

+ Present address: Soane Technologies, Inc., 3916 Trust Way, Hayward, CA 94545
} 
readily available data for thermodynamic properties such as vapor pressures, densities, and compressibilities. The new PHSC EOS is applied to LLE of binary blends containing polymers and copolymers and to binary LLE for solvent/polymer solutions.

We give attention here to LLE because representation of LLE is much more difficult than that of vapor-liquid equilibria (VLE). Several authors have shown that highly oversimplified models can represent VLE with one or two binary parameters, probably because VLE calculations require only the chemical potential of the solvent; because the polymer is not volatile, its chemical potential plays no role in VLE. However, in binary LLE chemical potentials of both components are important.

\section{NEW PERTURBED HARD-SPHERE-CHAIN EQUATION OF STATE}

Derivation of the PHSC EOS follows a first-order statistical-mechanical perturbation theory based on a fluid of hard-sphere chains as the reference system; details are given in our previous publications [6,7]. Here we only reproduce the main equations necessary for describing the model.

\subsection{Pure fluids}

The PHSC EOS for pure fluids is [6]

$$
\frac{p}{\rho k_{B} T}=1+r^{2} b \rho g\left(d^{+}\right)-(r-1)\left[g\left(d^{+}\right)-1\right]-\frac{r^{2} a \rho}{k_{B} T}
$$

where $p$ is the pressure, $\rho=N / N$ is the number density ( $N$ is the number of molecules and $V$ the volume), $k_{B}$ is the Boltzmann constant, $T$ is the absolute temperature, $g\left(d^{+}\right)$is the radial distribution function of hard spheres at contact, and $d$ is the effective hard-sphere diameter, $g\left(d^{+}\right)$is calculated from the Carnahan-Starling equation,

$$
g\left(d^{+}\right)=\frac{1-\eta / 2}{(1-\eta)^{3}} \quad \eta=\frac{r b p}{4}
$$

In Eq. (1), the first three terms represent the reference equation of state for hard-sphere chains, and the last term is a van der Waals-type perturbation to take into account attractive forces. The three segment-based parameters in Eq. (1), $r, b$, and $a$, all have a direct physical interpretation. Parameter $r$ represents the number of effective hard spheres per molecule. Parameter $b$ represents the second virial coefficient of hard spheres; it is an effective van der Waals covolume. Parameter $a$ reflects the strength of attractive forces between two non-bonded segments. In the PHSC theory, both $b$ and $a$ are temperature-dependent according to the Song-Mason method [10]:

$$
b(T)=\frac{2 \pi}{3} d^{3}(T)=\frac{2 \pi}{3} \sigma^{3} F_{b}\left(k_{B} T / \varepsilon\right) \quad a(T)=\frac{2 \pi}{3} \sigma^{3} \varepsilon F_{a}\left(k_{B} T / \varepsilon\right)
$$

where $\varepsilon$ and $\sigma$ are pair-potential parameters; $\varepsilon$ is the depth of the minimum in the pair potential and $\sigma$ is the separation distance between segment centers at this minimum. In Eq. (3), $F_{a}\left(k_{B} T / \varepsilon\right)$ and $F_{b}\left(k_{B} T / \varepsilon\right)$ are two universal functions of the reduced temperature, $k_{B} T / \varepsilon$. Originally, these two functions were determined from fitting the vapor pressures and saturated liquid and vapor densities for argon and methane by setting $r=1$.To apply original universal functions to molecular fluids $(r>1)$, parameter $s$ was introduced to rescale $F_{a}$ and $F_{b}$ with respect to temperature such that their values remained in a consistent range for both small and large molecules; $s$ is a function of molecular chain length only for pure fluids. However, the evaluation of $s$ in the mixture became uncertain since the definition of molecular chain length for cross terms was not clear and the inclusion of $s$ then led to arbitrary combining rules for mixtures.

In the present version of the PHSC EOS, scaling factor $s$ is removed and the two universal functions $F_{a}$ and $F_{b}$ are redetermined from thermodynamic properties of fluid argon and methane over large ranges of temperature and density; they are accurately represented by the following empirical formulae:

$$
\begin{aligned}
& F_{a}\left(k_{B} T / \varepsilon\right)=1.8681 \exp \left[-0.0619\left(k_{B} T / \varepsilon\right)\right]+0.6715 \exp \left[-1.7317\left(k_{B} T / \varepsilon\right)^{3 / 2}\right] \\
& F_{b}\left(k_{B} T / \varepsilon\right)=0.7303 \exp \left[-0.1649\left(k_{B} T / \varepsilon\right)^{1 / 2}\right]+0.2697 \exp \left[-2.3973\left(k_{B} T / \varepsilon\right)^{3 / 2}\right]
\end{aligned}
$$

Table 1 lists the new PHSC EOS parameters $r, \varepsilon, \sigma$ for normal fluids. The fitting procedure is the same as that used in the previous PHSC EOS [6] except that vapor pressures and saturated liquid densities at temperatures between $0.5 T_{c}$ and $0.9 T_{c}$ are used, rather than temperatures between the triple point and $0.9 T_{c}$. 


\subsection{Polymers}

Since $r$ is used as a measure of molecular size, for a polymer it is proportional to molecular weight $M$. Although both $r$ and $M$ are large for a polymer, the ratio $r / M$ remains constant as $M$ rises. Thus, for pure polymers $r / M$ (instead $r$ ) becomes a characteristic parameter in addition to $\varepsilon$ and $\sigma$. These three parameters for polymers can be determined from experimental $p V T$ data. For sufficiently high-molecular-weight polymers, $p V T$ data are usually insensitive to the molecular weight. This observation can be clearly seen by rewriting Eq. (1) as:

$\frac{p}{\rho_{r} k_{B} T}=1+b \rho_{r} g\left(d^{+}\right)-(1-1 / r) g\left(d^{+}\right)-\frac{a \rho_{r}}{k_{B} T}$

where $\rho_{r}=r \rho$ is the segment density of the polymer, which is the compatible quantity to the measured mass density of the polymer. In Eq. (6), $r$ appears explicitly only in the term (1-1/r), which becomes essentially unity for large $r$. Therefore, we can obtain a simpler EOS for polymers by taking the limit of $\mathrm{r} \rightarrow \infty$ in Eq. (6):

$\frac{p}{\rho_{r} k_{B} T}=1+b \rho_{r} g\left(d^{+}\right)-g\left(d^{+}\right)-\frac{a \rho_{r}}{k_{B} T}$

Non-linear least-squares regression is used to find a set of three molecular parameters $r / M, \varepsilon$ and $\sigma$ for each polymer by fitting Eq. (7) to $p V T$ data. Table 2 gives parameters regressed for a number of polymers.

\subsection{Mixtures containing polymers}

Extension of Eq. (1) to mixtures is straightforward [7]:

$$
\frac{p}{\rho k_{B} T}=1+\rho \sum_{i j}^{m} x_{i} x_{j} r_{i} r_{j} b_{i j} g_{i j}\left(d_{i j}^{+}\right)-\sum_{i}^{m} x_{i}\left(r_{i}-1\right)\left[g_{i i}\left(d_{i i}^{+}\right)-1\right]-\frac{\rho}{k_{B} T} \sum_{i j}^{m} x_{i} x_{j} r_{i} r_{j} a_{i j}
$$

where $x_{i}=N / N$ is the number fraction of molecules, $r_{i}$ is the number of segments (tangent hard spheres) comprising component $i=1,2, \ldots, m$, and $g_{i j}\left(d_{i j}{ }^{+}\right)$is the $i j$ pair radial distribution function of hard-sphere mixtures at contact. For each unlike pair of components $(i \neq j)$, additional parameters, $b_{i j}$ and $a_{i j}$, are needed for the mixture; $b_{i j}$ is the second cross virial coefficient of hard-sphere mixtures and $a_{i j}$, is the parameter reflecting attractive forces between two unlike non-bonded segments. A combining rule is not necessary for calculating $b_{i j}$ because hard-sphere diameters are additive:

$$
b_{i j}(T)=\frac{2 \pi}{3} d_{i j}^{3}(T)=\left(b_{i}^{1 / 3}+b_{j}^{1 / 3}\right)^{3} / 8 \quad d_{i j}(T)=\left[d_{i}(T)+d_{j}(T)\right] / 2
$$

However, this restriction can be relaxed when applying Eq. (9) to real mixtures; an alternate expression for $b_{i j}$ and $a_{i j}$ can be obtained by extending Eq. (3) to mixtures:

$$
\begin{array}{ll}
b_{i j}(T)=\frac{2 \pi}{3} d_{i j}^{3}(T)=\frac{2 \pi}{3} \sigma_{i j}^{3} F_{b}\left(k_{B} T / \varepsilon_{i j}\right) & a_{i j}(T)=\frac{2 \pi}{3} \sigma_{i j}^{3} \varepsilon_{i j} F_{a}\left(k_{B} T / \varepsilon_{i j}\right) \\
\sigma_{i j}=\frac{1}{2}\left(\sigma_{i}+\sigma_{j}\right)\left(1-\lambda_{i j}\right) & \varepsilon_{i j}=\left(\varepsilon_{i j} \varepsilon_{j j}\right)^{4 / 2}\left(1-\kappa_{i j}\right)
\end{array}
$$

where $\lambda_{i j}$ and $\kappa_{i j}$ are adjustable binary parameters. Finally, $g_{i j}\left(d_{i j}{ }^{+}\right)$is given by

$$
\begin{aligned}
& g_{i j}\left(d_{i j}^{+}\right)=\frac{1}{1-\eta}+\frac{3}{2} \frac{\xi_{i j}}{(1-\eta)^{2}}+\frac{1}{2} \frac{\xi_{i j}^{2}}{(1-\eta)^{3}} \\
& \eta=\frac{\rho}{4} \sum_{i}^{m} x_{i} r_{i} b_{i} \\
& \xi_{i j}=\frac{p}{4}\left(b_{i} b_{j} / b_{i j}\right)^{1 / 3} \sum_{i}^{m} x_{k} r_{k} b_{k}^{2 / 3}
\end{aligned}
$$

\section{EXTENSION TO COPOLYMER SYSTEMS}

An important advantage of the PHSC theory is that segments (spheres) in a chain molecule need not all have the same diameter. Therefore, the PHSC EOS can be used to represent copolymers and their mixtures where different segments have different diameters.

\subsection{Pure copolymers}

Considering a pure copolymer system where each molecule consists of $r$ segments but each segment has a different size, the PHSC EOS is [9] 
Table 1

PHSC equation-of-state parameters for some normal fluids

\begin{tabular}{|c|c|c|c|c|c|c|c|}
\hline Substance & $r$ & $\varepsilon / k_{B}(\mathrm{~K})$ & $\sigma(\AA)$ & Substance & $r$ & $\varepsilon / k_{B}(\mathrm{~K})$ & $\sigma(\AA)$ \\
\hline \multicolumn{4}{|l|}{ normal alkanes } & \multicolumn{4}{|l|}{ aromatics } \\
\hline ethane & 1.694 & 206.3 & 3.916 & benzene & 2.727 & 291.6 & 3.958 \\
\hline propane & 2.129 & 219.0 & 3.998 & toluene & 3.138 & 287.0 & 4.019 \\
\hline butane & 2.496 & 231.3 & 4.085 & ethylbenzene & 3.607 & 279.4 & 4.018 \\
\hline pentane & 3.149 & 226.0 & 3.995 & propylbenzene & 4.137 & 270.6 & 4.007 \\
\hline hexane & 3.446 & 235.6 & 4.084 & o-xylene & 3.620 & 285.4 & 3.998 \\
\hline heptane & 4.255 & 225.9 & 3.947 & m-xylene & 3.721 & 276.4 & 3.977 \\
\hline octane & 5.055 & 219.6 & 3.850 & p-xylene & 3.455 & 287.9 & 4.104 \\
\hline nonane & 5.748 & 217.3 & 3.804 & chlorobenzene & 3.144 & 305.3 & 3.975 \\
\hline decane & 6.616 & 212.7 & 3.723 & fiuorobenzene & 3.012 & 276.9 & 3.883 \\
\hline undecane & 7.057 & 215.4 & 3.754 & styrene & 3.291 & 304.3 & 4.083 \\
\hline dodecane & 7.712 & 214.8 & 3.733 & & & & \\
\hline tridecane & 7.986 & 218.3 & 3.794 & \multicolumn{4}{|l|}{ acetates } \\
\hline tetradecane & 9.023 & 213.7 & 3.682 & methyl acetate & 3.804 & 224.8 & 3.338 \\
\hline pentadecane & 9.851 & 211.3 & 3.662 & ethyl acetate & 4.509 & 213.5 & 3.369 \\
\hline hexadecane & 10.168 & 214.2 & 3.703 & n-propyl acetate & 4.989 & 213.9 & 3.440 \\
\hline heptadecane & 10.834 & 213.7 & 3.680 & i-propyl acetate & 4.779 & 210.6 & 3.484 \\
\hline octadecane & 11.110 & 216.5 & 3.698 & n-butyl acetate & 5.413 & 216.3 & 3.514 \\
\hline nonadecane & 11.659 & 216.1 & 3.718 & i-butyl acetate & 5.574 & 207.4 & 3.461 \\
\hline \multirow[t]{2}{*}{ eicosane } & \multirow{2}{*}{12.925} & \multirow{3}{*}{212.1} & \multirow{3}{*}{3.633} & t-butyl acetate & 4.485 & 220.2 & 3.743 \\
\hline & & & & n-pentyl acetate & 6.547 & 206.6 & 3.403 \\
\hline \multicolumn{2}{|c|}{ branched \& cyclic alkanes } & & & i-pentyl acetate & 5.568 & 220.5 & 3.628 \\
\hline isobutane & 2.397 & 226.2 & 4.155 & n-hexyl acetate & 6.720 & 212.9 & 3.514 \\
\hline isopentane & 2.777 & 236.7 & 4.182 & vinyl acetate & 4.259 & 218.2 & 3.371 \\
\hline neopentane & 2.526 & 233.7 & 4.351 & & & & \\
\hline 2,2-dimethylbutane & 2.849 & 247.7 & 4.368 & \multicolumn{4}{|l|}{ ethers } \\
\hline 2,3-dimethylbutane & 2.998 & 247.1 & 4.278 & dimethyl ether & 2.519 & 216.6 & 3.553 \\
\hline cyclopentane & 2.509 & 277.3 & 4.090 & diethyl ether & 2.985 & 237.9 & 3.780 \\
\hline methylcyclopentane & 2.902 & 267.2 & 4.153 & ethylene oxide & 2.242 & 277.5 & 3.390 \\
\hline ethylcyclopentane & 3.277 & 269.4 & 4.181 & 1,3-propylene oxide & 2.381 & 295.4 & 3.729 \\
\hline propylcyclopentane & 3.823 & 262.7 & 4.130 & tetrahydrofiuran & 2.758 & 279.5 & 3.811 \\
\hline cyclohexane & 2.723 & 286.7 & 4.215 & & & & \\
\hline methylcyclohexane & 2.968 & 283.7 & 4.336 & \multicolumn{4}{|l|}{ ketones } \\
\hline ethylcyclohexane & 3.392 & 282.4 & 4.306 & acetone & 3.164 & 250.9 & 3.510 \\
\hline propylcyclohexane & 3.969 & 272.3 & 4.230 & methyl ethyl ketone & 3.344 & 255.8 & 3.694 \\
\hline \multirow[t]{2}{*}{ butylcyclohexane } & 4.580 & 264.5 & 4.151 & diethyl ketone & 3.931 & 245.4 & 3.684 \\
\hline & & & & 2-pentanone & 4.041 & 241.2 & 3.661 \\
\hline alkenes & & & & 2-bexanone & 5.151 & 224.5 & 3.506 \\
\hline ethylene & 1.609 & 196.8 & 3.839 & & & & \\
\hline propylene & 2.029 & 221.9 & 3.951 & \multicolumn{4}{|c|}{ chlorinated hydrocarbons } \\
\hline 1-butene & 2.677 & 218.3 & 3.882 & methyl chloride & 2.018 & 256.7 & 3.591 \\
\hline trans-2-butene & 2.504 & 233.9 & 3.984 & dichloromethane & 2.669 & 268.1 & 3.541 \\
\hline cis-2-butene & 2.530 & 235.7 & 3.941 & carbon tetrachloride & 2.507 & 301.7 & 4.187 \\
\hline 1-pentene & 2.828 & 237.1 & 4.087 & chloroform & 2.878 & 268.1 & 3.726 \\
\hline 1-hexene & 3.395 & 234.5 & 4.042 & vinyl chloride & 2.175 & 249.7 & 3.773 \\
\hline trans-decalin & 3.160 & 333.8 & 4.657 & 1,2-dichlorethane & 2.994 & 280.9 & 3.691 \\
\hline
\end{tabular}

Table 2

PHSC equation-of-state parameters for common polymers

\begin{tabular}{|c|c|c|c|c|c|c|c|}
\hline Polymer & $r / M$ & $\varepsilon / k_{B}(\mathrm{~K})$ & $\sigma(\AA)$ & Polymer & $r / M$ & $\varepsilon / k_{B}(\mathrm{~K})$ & $\sigma(\AA)$ \\
\hline high density polyethylene & 0.04938 & 324.1 & 3.825 & poly(vinyl acetate) & 0.05166 & 292.6 & 3.346 \\
\hline low density polyethylene & 0.05854 & 306.0 & 3.603 & poly (methyl methacrylate) & 0.04222 & 373.5 & 3.608 \\
\hline$i$-poly(propylene) & 0.02831 & 392.1 & 4.705 & $i$-poly(methyl methacrylate) & 0.05071 & 347.6 & 3.367 \\
\hline i-poly(1-butene) & 0.04024 & 353.2 & 4.120 & poly(butyl methacrylate) & 0.04764 & 323.4 & 3.604 \\
\hline poly(isobutene) & 0.04963 & 331.9 & 3.725 & poly (cyclohexyl methactylate) & 0.03742 & 379.3 & 3.856 \\
\hline poly(4-methyl-1-pentene) & 0.02890 & 381.7 & 4.685 & poly(ethylene terephthalate) & 0.04777 & 363.5 & 3.320 \\
\hline poly(styrene) & 0.03834 & 385.4 & 3.899 & poly(carbonate) & 0.03885 & 393.0 & 3.707 \\
\hline poly(o-methyl styrene) & 0.03925 & 393.5 & 3.893 & poly(ether ether ketone) & 0.04424 & 375.0 & 3.429 \\
\hline cis-1,4-poly(butadiene) & 0.07049 & 276.8 & 3.301 & poly(sulphone) & 0.03720 & 425.7 & 3.721 \\
\hline poly(vinyl chloride) & 0.03672 & 365.1 & 3.590 & poly(tetrafiuoroethylene) & 0.02705 & 215.9 & 3.244 \\
\hline poly(ethylene glycol) & 0.04778 & 316.6 & 3.532 & poly(tetrahydrofuran) & 0.04277 & 327.6 & 3.833 \\
\hline
\end{tabular}




$$
\frac{p}{\rho k_{B} T}=1+\rho \sum_{\alpha=1}^{r} \sum_{\beta=1}^{r} b_{\alpha \beta} g_{\alpha \beta}-\sum_{\alpha=1}^{r-1}\left[g_{\alpha, \alpha+1}-1\right]-\frac{\rho}{k_{B} T} \sum_{\alpha=1}^{r} \sum_{\beta=1}^{r} a_{\alpha \beta}
$$

where subscripts $\alpha$ and $\beta$ denote the $\alpha$ th and $\beta$ th segments (effective hard spheres) in a copolymer molecule, $b_{\alpha \beta}$ is the van der Waals covolume between two segments, $g_{\alpha \beta}$ is the radial distribution function of hard spheres when the $\alpha$ th and $\beta$ th segments are at contact, and $a_{\alpha \beta}$ is the parameter reflecting attractive forces between non-bonded segments $\alpha$ and $\beta$. Parameters $b_{\alpha \beta}, g_{\alpha \beta}$ and $a_{\alpha \beta}$ are given by

$$
\begin{aligned}
& b_{\alpha}(T)=\frac{2 \pi}{3} \sigma_{\alpha}^{3} F_{b}\left(k_{B} T / \varepsilon_{\alpha \alpha}\right) \quad b_{\alpha \beta}(T)=\frac{1}{8}\left(b_{\alpha}^{1 / 3}+b_{\beta}^{1 / 3}\right)^{3} \\
& g_{\alpha \beta}=\frac{1}{1-\eta}+\frac{3}{2} \frac{\xi_{\alpha \beta}}{(1-\eta)^{2}}+\frac{1}{2} \frac{\xi_{\alpha \beta}^{2}}{(1-\eta)^{3}} \\
& \eta=\frac{\rho}{4} \sum_{\alpha=1}^{r} b_{\alpha} \quad \xi_{\alpha \beta}=\frac{\rho}{4}\left(b_{\alpha} b_{\beta} / b_{\alpha \beta}\right)^{1 / 3} \sum_{\gamma=1}^{r} b_{\gamma}^{2 / 3} \\
& a_{\alpha \beta}(T)=\frac{2 \pi}{3} \sigma_{\alpha \beta}^{3} \varepsilon_{\alpha \beta} F_{a}\left(k_{B} T / \varepsilon_{\alpha \beta}\right) \quad \sigma_{\alpha \beta}=\left(\sigma_{\alpha}+\sigma_{\beta}\right) / 2 \quad \varepsilon_{\alpha \beta}=\left(\varepsilon_{\alpha \alpha} \varepsilon_{\beta \beta}\right)^{1 / 2}\left(1-\kappa_{\alpha \beta}\right)
\end{aligned}
$$

where $\kappa_{\alpha \beta}$ is an adjustable parameter.

\subsection{Mixtures containing copolymers}

Equation (14) is readily extended to mixtures containing copolymers:

$$
\frac{p}{\rho k_{B} T}=1+\rho \sum_{i j}^{m} x_{i} x_{j}\left[\sum_{\alpha=1}^{r_{i}} \sum_{\beta=1}^{r_{j}} b_{i j, \alpha \beta} g_{i j, \alpha \beta}\right]-\sum_{i}^{m} x_{i} \sum_{\alpha=1}^{r_{i}}\left[g_{i i, \alpha, \alpha+1}-1\right]-\frac{\rho}{k_{B} T} \sum_{i j}^{m} x_{i} x_{j}\left[\sum_{\alpha=1}^{r_{i}} \sum_{\beta=1}^{r_{j}} a_{i j, \alpha \beta}\right]
$$

where indices $i$ and $j$ denote components in the mixture; they should not be confused with indices $\alpha$ and $\beta$ labeling segments in a molecule. The physical significances of $b_{i j, \alpha \beta}, g_{i j, \alpha \beta}$ and $a_{i j, \alpha \beta}$ are the same as those for pure fluids in Eq. (14). We again have two choices for calculating $b_{i j, \alpha \beta}$, one follows from hard-sphere additivity:

$b_{i j, \alpha \beta}(T)=\frac{2 \pi}{3} d_{i j, \alpha \beta}^{3}(T)=\frac{1}{8}\left(b_{i, \alpha}^{1 / 3}+b_{j, \beta}^{1 / 3}\right)^{3} \quad d_{i j, \alpha \beta}(T)=\left[d_{i, \alpha}(T)+d_{j, \beta}(T)\right] / 2$

Another method for $b_{i j, \alpha \beta}$ and $a_{i j}, \alpha \beta$ can be obtained by extending Eq. (10) to copolymer mixtures:

$$
\begin{array}{ll}
b_{i j, \alpha \beta}(T)=\frac{2 \pi}{3} d_{i j, \alpha \beta}^{3}(T)=\frac{2 \pi}{3} \sigma_{i j, \alpha \beta}^{3} F_{b}\left(k_{\beta} T / \varepsilon_{i j, \alpha \beta}\right) & a_{i j, \alpha \beta}(T)=\frac{2 \pi}{3} \sigma_{i j, \alpha \beta}^{3} \varepsilon_{i j, \alpha \beta} F_{a}\left(k_{B} T / \varepsilon_{i j, \alpha \beta}\right) \\
\sigma_{i j, \alpha \beta}=\frac{1}{2}\left(\sigma_{i, \alpha}+\sigma_{j, \beta}\right)\left(1-\lambda_{i j, \alpha \beta}\right) & \varepsilon_{i j, \alpha \beta}=\left(\varepsilon_{i i, \alpha \alpha \beta} \varepsilon_{i j, \beta \beta}\right)^{1 / 2}\left(1-\kappa_{i j, \alpha \beta}\right)
\end{array}
$$

where $\lambda_{i j, \alpha \beta}$ and $\kappa_{i j, \alpha \beta}$ are adjustable parameters. Finally, $g_{i j, \alpha \beta}$ is given by

$$
\begin{array}{ll}
g_{i j, \alpha \beta}=\frac{1}{1-\eta}+\frac{3}{2} \frac{\xi_{i j, \alpha \beta}}{(1-\eta)^{2}}+\frac{1}{2} \frac{\xi_{i j, \alpha \beta}^{2}}{(1-\eta)^{3}} & \\
\eta=\frac{\rho}{4} \sum_{i}^{m} x_{i}\left[\sum_{\alpha=1}^{r_{i}} b_{i, \alpha}\right] & \xi_{i j, \alpha \beta}=\frac{\rho}{4}\left(b_{i, \alpha} b_{j, \beta} / b_{i j, \alpha \beta}\right)^{1 / 3} \sum_{k}^{m} x_{k}\left[\sum_{\gamma=1}^{r_{k}} b_{k, \gamma}^{2 / 3}\right]
\end{array}
$$

\section{COMPARISON WITH EXPERIMENT}

To correlate experimental LLE, it is necessary to use Eqs. (10) and (11) to calculate $b_{i j}(i \neq j)$ and $a_{i j}(i \neq j)$ for mixtures containing polymers and to use Eqs. (21) and (22) to calculate $b_{i j, \alpha \beta}(i \neq j)$ and $a_{i j, \alpha \beta}$ ( $i \neq j$ ) for mixtures containing copolymers. To illustrate, Figure 1 compares theoretical coexistence curves with experimental data [11] for poly $(\alpha$-methyl styrene)(PMS)/polystyrene(PS) mixtures which exhibit UCST behavior. The EOS parameters for poly (o-methyl styrene) are those for poly ( $\alpha$-methyl styrene) (see Table 2$)$. Binary parameters $\lambda_{l 2}$ $(0.0000924)$ and $\kappa_{12}(-0.0000585)$ are determined such that theory agrees with experiment for both PMS62/PS58 and PMS56/PS49 (where PMS62, e.g., denotes PMS at the weight-average molecular weight 62000). Agreement is good but sensitive to the choice of binary parameters. The semibroken line is the glass-transition temperature $T_{g}$ of the mixture, calculated from this equation: $1 / T_{g}(K)=w_{P S} / 373+\left(1-w_{P S}\right) / 444$ where $w_{P S}$ is the weight fraction of 
polystyrene. The PHSC theory predicts that the coexistence curve of PMS44/PS37 lies below the glass-transition temperature of the mixture, consistent with experimental observation [11].

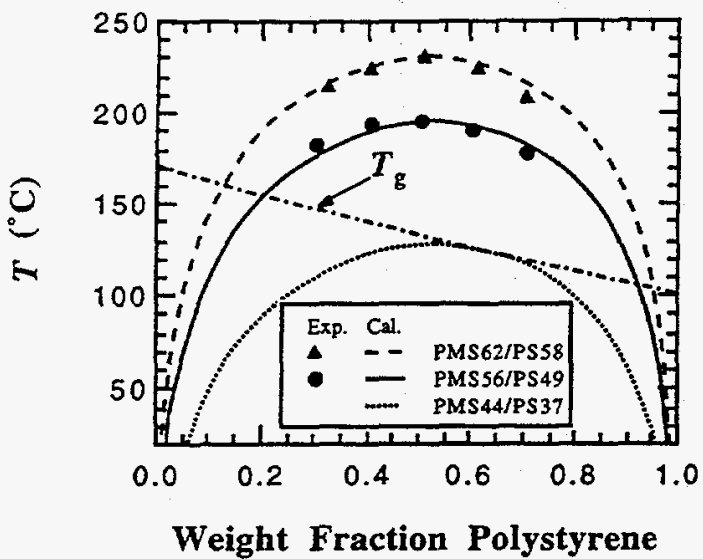

Fig.1. Coexistence curve for PMS/PS mixtures

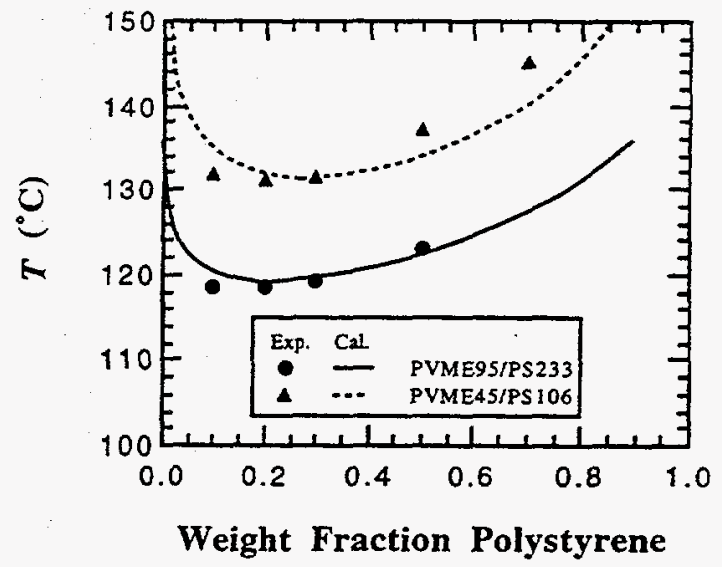

Fig.2. Coexistence Curve for PVME/PS mixtures

Figure 2 compares theoretical coexistence curves with experiment for poly(vinyl methyl ether)(PVME)/polystyrene mixtures which exhibit LCST behavior. Experimental data are from Walsh et al. [12]. Binary parameters $\lambda_{l 2}(0.000152)$ and $\kappa_{12}(0.000720)$ are determined as in Figure 1. Agreement is good although the PHSC theory slightly overestimates the critical weight fraction of polystyrene.

Figure 3 compares a theoretical miscibility map with experiment for binary mixtures of poly(styrene-comethyl methacrylate) (S-co-MMA) random copolymers differing in copolymer compositions [13]. The weightaverage molecular weight of copolymer sample is 150000 and parameters $\lambda_{\text {S-MMA }}(-0.013)$ and $\kappa_{\text {S-MMA }}(0.01626)$ are determined by fitting to experimental data. In this mixture, the only difference between components 1 and 2 is in copolymer composition; the mixture becomes more miscible as the difference in copolymer composition declines. Therefore, the miscibility map shown in Figure 3 has a miscible region around the diagonal line. The miscible area decreases with temperature because the miscible pair of copolymers eventually exhibits phase separation at elevated temperature due to LCST behavior. Although it is difficult to identify the miscibilityimmiscibility boundary from experimental data, theory and experiment appears to be in fair agreement.

Theoretical LLE for solvent/polymer systems (where molecules are significantly different in size) show deviations from experiment; the PHSC EOS overestimates the EOS effect which causes the LCST at elevated temperature[14]. As a result, the PHSC EOS predicts a LCST lower than experiment when pure-component parameters of polymers are used. To improve agreement, we introduce an adjustable parameter that, in effect, decreases densities of pure polymers [14]; here we present only a brief summary and give an example.

Consider a binary solvent/polymer system where components 1 and 2 represent solvent and polymer, respectively. In Eq. (8), the term $r_{i} r_{a_{i j}}$ in the perturbation term represents the attractive force between components $i$ and $j$. The density of a polymer can be decreased by decreasing the magnitude of $r_{2} r_{2} a_{22}$ in the perturbation term. Therefore, we correct the overestimated EOS effect in dilute polymer solutions by multiplying $r_{2}$ by a factor $\zeta$ smaller than unity in the perturbation term [14]. By introducing adjustable parameter $\zeta$, we require that hardsphere diameters of unlike segments are additive; i.e., $b_{22}$ is given by Eq. (9). As a result, we use only two adjustable parameters $\zeta$ and $\kappa_{12}$ for binary mixtures.

Figure 4 shows the effect of molecular weight on LCST and UCST for $n$-pentyl acetate $(n$ $\left.\mathrm{CH}_{3} \mathrm{CO}_{2} \mathrm{C}_{5} \mathrm{H}_{11}\right) /$ polyethylene(PE) mixtures [15]. Here $r=r_{2} / r_{1}$ and the molecular-weight range of polyethylene is $M_{\eta}=13600-175000$ where $M_{\eta}$ is the viscosity-average molecular weight. Two adjustable parameters are: $\zeta=0.824$ and $\kappa_{12}=0.01777$. The mixture is miscible in all proportions at temperatures between LCST and UCST. The PHSC EOS is able to represent simultaneous occurrence of an UCST and a LCST in this solventpolymer system. 


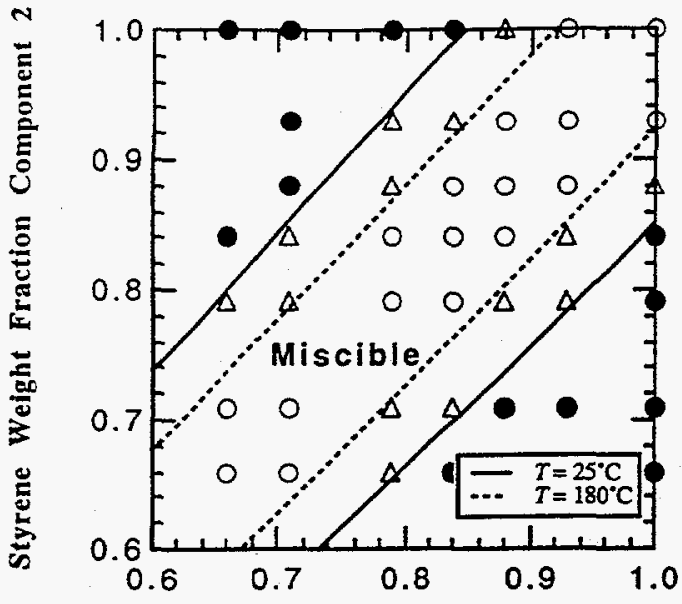

Styrene Weight Fraction Component 1

Fig.3. Miscibility Map for P(S-co-MMA) Copolymer Mixtures

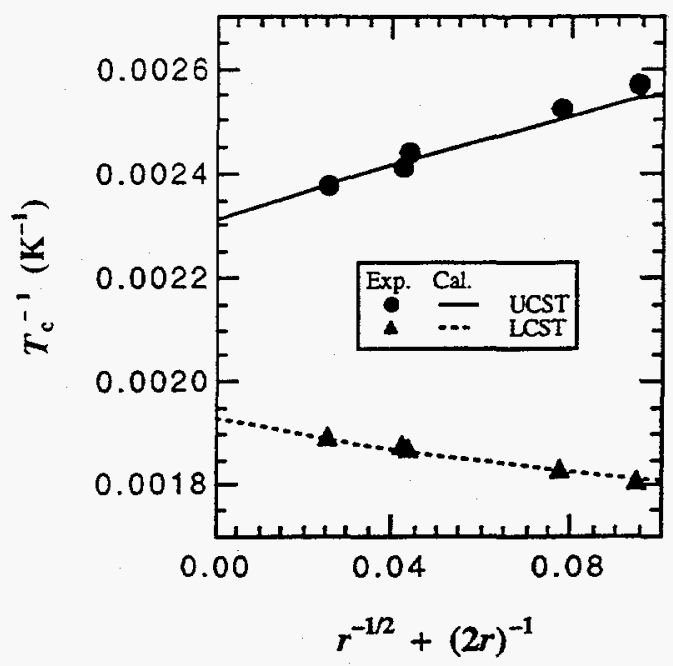

Fig.4. Molecular-Weight Dependence of LCST UCST for $n-\mathrm{CH}_{3} \mathrm{CO}_{2} \mathrm{C}_{5} \mathrm{H}_{11} / \mathrm{PE}$ mixtures

\section{CONCLUSION}

A simplified PHSC EOS is presented and applied to LLE of binary mixtures containing solvents, polymers, and copolymers. The PHSC EOS is able to represent UCST behavior as well as LCST behavior in polymer blends including copolymer mixtures. Theoretical and experimental coexistence curves and miscibility maps show good agreement for mixtures containing poly( $\alpha$-methyl styrene), poly(styrene), poly(vinyl methyl ether), and poly(styrene-co-methyl methacrylate). To correct the overestimated EOS effect in solvent/polymer systems, an empirical correction is introduced into the perturbation term of the PHSC EOS to reduce the polymer's chain length. This correction is probably necessary to compensate for failure of the mean-field assumption for dilute and semi-dilute polymer solutions. The modified PHSC EOS can quantitatively represent simultaneous occurrence of UCST and LCST in selected solvent/polymer systems, including molecular-weight dependence of both critical solution temperatures.

\section{ACKNOWLEDGMENT}

This work is supported by the Director, Office of Energy Research, Office of Basic Energy Sciences, Chemical Sciences Division of the U.S. Department of Energy, under Contact No. DE-AC03-76SF00098. Additional funding was provided by E.I. du Pont Nemours \& Co. (Philadelphia, PA) and Koninklijke Shell (Amsterdam, The Netherlands).

\section{REFERENCES}

1. P.J. Flory, R.A. Orwoll, and A. Vrij, JACS, 86, 3507 (1964).

2. D. Patterson and G. Delmas, Discuss. Faraday Soc., 49, 98 (1970).

3. P.J. Flory, Principles of Polymer Chemistry. 1953, Ithaca, N.Y.: Cornell University Press.

4. Y. Song, S.M. Lambert, and J.M. Prausnitz, presented at AIChE Annual meeting, St. Louis, MO, 1993.

5. Y. Song, S.M. Lambert, and J.M. Prausnitz, Macromolecules, 27, 441 (1994).

6. Y. Song, S.M. Lambert, and J.M. Prausnitz, Ind. \& Eng. Chem. Res., 33, 1047 (1994).

7. Y. Song, S.M. Lambert, and J.M. Prausnitz, Chem. Eng. Sci., 49, 2765 (1994).

8. Y. Song, S.M. Lambert, and J.M. Prausnitz, presented at AIChE Annual Meeting, San Francisco, CA, 1994.

9. T. Hino, Y. Song, and J.M. Prausnitz, Macromolecules, 27, 5681 (1994).

10. Y. Song and E.A. Mason, J. Chem. Phys., 91, 7840 (1989).

11. J.L. Lin and R.J. Roe, Polymer, 29, 1227 (1988).

12. D.J. Walsh, G.T. Dee, J.L. Halary, J.M. Ubiche, M. Millequant, and L. Monnerie, Macromolecules, 22, 3395 (1989).

13. D. Braun, D. Yu, P.R. Kohl, X. Gao, L.N. Andradi, and G.P. Hellmann, J. Polym. Sci.: Polym. Phys., 30, 577 (1992).

14. T. Hino, Y. Song, and J.M. Prausnitz, Macromolecules, to be submitted (1995)

15. N. Kuwahara, S. Saeki, T. Chiba, and M. Kaneko, Polymer, 15, 777 (1974). 